Original paper

\title{
Proton range monitoring with in-beam PET: Monte Carlo activity predictions and comparison with cyclotron data
}

\author{
A.C. Kraan ${ }^{\mathrm{a}, \mathrm{b}, *}$, G. Battistoni ${ }^{\mathrm{c}}$, N. Belcari ${ }^{\mathrm{a}, \mathrm{b}}$, N. Camarlinghi ${ }^{\mathrm{a}, \mathrm{b}}$, G.A.P. Cirrone ${ }^{\mathrm{d}}$, G. Cuttone ${ }^{\mathrm{d}}$, \\ S. Ferretti ${ }^{\mathrm{a}, \mathrm{b}}$, A. Ferrari ${ }^{\mathrm{e}}$, G. Pirrone ${ }^{\mathrm{a}, \mathrm{b}}$, F. Romano $^{\mathrm{d}}$, P. Sala ${ }^{\mathrm{c}}$, G. Sportelli $^{\mathrm{a}, \mathrm{b}}$, K. Straub ${ }^{\mathrm{a}, \mathrm{b}}$, \\ A. Tramontana ${ }^{\mathrm{d}, \mathrm{f}}, \mathrm{A}$. Del Guerra ${ }^{\mathrm{a}, \mathrm{b}}, \mathrm{V}$. Rosso ${ }^{\mathrm{a}, \mathrm{b}}$ \\ ${ }^{a}$ Department of Physics, University of Pisa, Pisa, Italy \\ ${ }^{\mathrm{b}}$ INFN, Pisa, Italy \\ ' INFN Sezione di Milano, Milano, Italy \\ ${ }^{\mathrm{d}}$ INFN-Laboratori Nazionali del Sud, Catania, Italy \\ ${ }^{\text {e } C E R N, \text { Geneva, Switzerland }}$ \\ ${ }^{\mathrm{f}}$ Department of Physics and Astronomy, University of Catania, Catania, Italy
}

\section{A R T I C L E I N F O}

\section{Article history:}

Received 19 January 2014

Received in revised form 3 April 2014

Accepted 6 April 2014

Available online $\mathrm{xxx}$

\section{Keywords:}

Hadron therapy

In-beam PET

Treatment quality monitoring

Cyclotron

\begin{abstract}
A B S T R A C T
Goal: Proton treatment monitoring with Positron-Emission-Tomography (PET) is based on comparing measured and Monte Carlo (MC) predicted $\beta^{+}$activity distributions. Here we present PET $\beta^{+}$activity data and MC predictions both during and after proton irradiation of homogeneous PMMA targets, where protons were extracted from a cyclotron.

Methods and materials: PMMA phantoms were irradiated with $62 \mathrm{MeV}$ protons extracted from the CATANA cyclotron. PET activity data were acquired with a $10 \times 10 \mathrm{~cm}^{2}$ planar PET system and compared with predictions from the FLUKA MC generator. We investigated which isotopes are produced and decay during irradiation, and compared them to the situation after irradiation. For various irradiation conditions we compared one-dimensional activity distributions of MC and data, focussing on $\Delta \mathrm{w} 50 \%$, i.e., the distance between the $50 \%$ rise and $50 \%$ fall-off position.

Results: The PET system is able to acquire data during and after cyclotron irradiation. For PMMA phantoms the difference between the FLUKA MC prediction and our data in $\Delta \mathrm{w} 50 \%$ is less than $1 \mathrm{~mm}$. The ratio of PET activity events during and after irradiation is about 1 in both data and FLUKA, when equal time-frames are considered. Some differences are observed in profile shape.

Conclusion: We found a good agreement in $\Delta \mathrm{w} 50 \%$ and in the ratio between beam-on and beam-off activity between the PET data and the FLUKA MC predictions in all irradiation conditions.
\end{abstract}

() 2014 Associazione Italiana di Fisica Medica. Published by Elsevier Ltd. All rights reserved.

\section{Introduction}

Radiotherapy plays an important role in modern cancer treatment, with about $50 \%$ of all cancer patients receiving radiotherapy [1]. The main challenge in radiotherapy is how to deliver high dose to the tumour region, while minimizing dose to healthy tissue. Proton therapy is a promising radiotherapy technique, because it offers the possibility to deliver high dose in well-defined volumes (Bragg-peak). However, the steep dose gradients make proton therapy much more sensitive to treatment uncertainties than

\footnotetext{
* Corresponding author. INFN, Edificio C - Polo Fibonacci Largo B. Pontecorvo, 3 - 56127 Pisa, Italy. Tel.: +39 0502214414

E-mail addresses: aafke.kraan@pi.infn.it, aafkekraan@gmail.com (A.C. Kraan).
}

conventionally used X-ray therapy. Indeed, uncertainties in patient positioning, proton range and anatomical changes can cause dose distortions, possibly impairing the beneficial effects of charged particle therapy.

For this reason, it is highly desirable to monitor the effectively delivered dose, or at least the particle range in patients. PET imaging is a non-invasive way of in-vivo verification of the dose delivered to the target volume. During ion beam irradiation, various $\beta^{+}$emitting isotopes $\left({ }^{15} \mathrm{O},{ }^{11} \mathrm{C},{ }^{13} \mathrm{~N}\right.$, etc $)$ are generated in the patient. These $\beta^{+}$annihilations can be detected with a PET system during or after the irradiation, depending on the half-life of the $\beta^{+}$emitting isotope. Since dose and $\beta^{+}$activity result from different physics processes, the relation between them is indirect, as shown in Fig. 1. By measuring the $\beta^{+}$activity in a certain time-frame, and by comparing it to planned $\beta^{+}$activity from Monte Carlo simulations, 


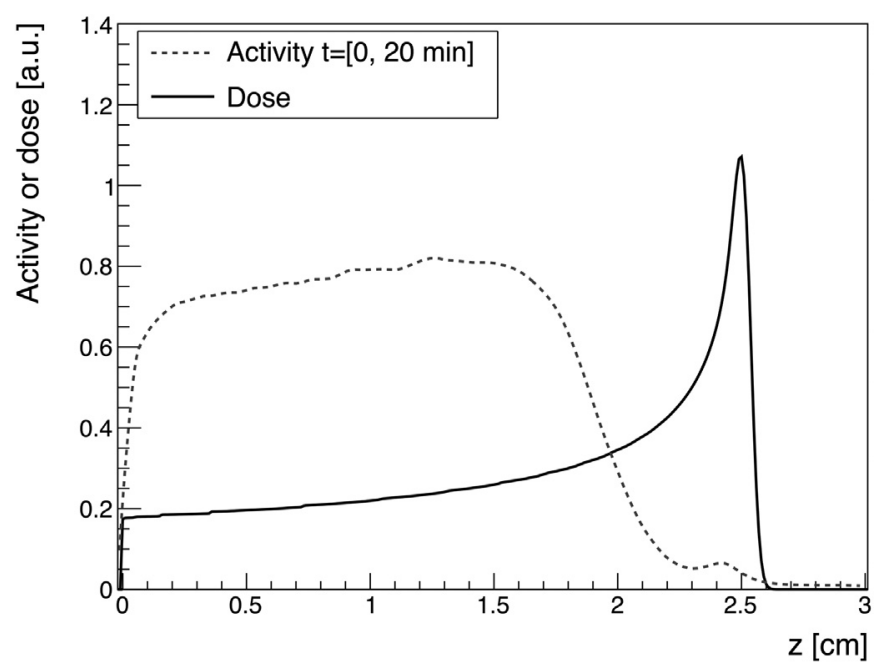

Figure 1. Simulated Bragg peak and activity 1-D profile along the $z$-direction (beam direction) of $58 \mathrm{MeV}$ protons on a PMMA target, obtained with a FLUKA Monte Carlo simulation of $800 \mathrm{M}$ protons.

it is possible to verify whether the dose was delivered correctly. If large differences are found between the measured and planned $\beta^{+}$ activity distributions, the treatment can be adjusted. For more details about in-vivo proton range verification we refer to recent review papers by Knopf and Lomax [2] and Zhu and El Fahkri [3].

There are different PET data-taking strategies. In 'offline PET data acquisition', data are acquired after patient irradiation with a commercial PET-CT scanner outside the treatment room, and PET data are usually acquired after a CT scan. Although the method is economically attractive, biological washout and patient movement limit the use of this method. Essentially only the $\beta^{+}$activity from ${ }^{11} \mathrm{C}$ (half-life $20 \mathrm{~min}$ ) can be detected. This method has been clinically applied in several treatment centres, see for instance references [4-8]. Another strategy is so-called 'in-room PET data acquisition'. Here a full-ring PET detector is installed inside the treatment room $[9,10]$. The main advantage with respect to offline imaging is that signal washout is greatly reduced, as it allows for detection of activity from ${ }^{15} \mathrm{O}$ (half-life $2 \mathrm{~min}$ ). This isotope is produced in abundance in human tissue during irradiation. Also, no repositioning of the patient is necessary. Disadvantages include a slower patient throughput and problems with co-registration of PET and CT images. Another promising strategy is 'in-beam PET data acquisition'. Here a PET detector is integrated in the beam-delivery system [11-15]. The advantage is that data can be taken not only after, but also during irradiation, so problems related to washout and patient motion are minimized. One of the main technical difficulties is the integration into the beam delivery system. Dual-head PET systems $[13,15,16]$ are relatively easy to install, but have limited angular coverage, resulting in low sensitivity and artifacts in reconstructed images. Time-of-flight techniques are proposed to counterbalance these issues [17]. PET systems with more efficient geometries have been developed and include a dual-ring [18] and a full-ring [19] PET, cut at a slant angle. Apart from geometrical issues, another important challenge of in-beam PET is to take advantage of the full irradiation time interval, i.e., to include not only data acquired after irradiation of during beam-pauses, but also during beam extraction [20]. In fact, background from random coincidences tends to paralyze the PET detectors, and advanced techniques are required for background suppression [21].

In this last context, a compact planar PET prototype has been developed and built in Pisa, which can be installed in the beam delivery system. This system is capable of acquiring data during ('beam-on') and after ('beam-off') particle irradiation, as was demonstrated recently [15,22] for proton irradiation with the CATANA (Center for Hadron Therapy and Advanced Nuclear Applications, Centro di AdroTerapia e Applicazioni Nucleari Avanzate) cyclotron. Including data acquired during irradiation was seen to improve the quality of range measurements in PMMA with respect to data acquired only after irradiation. It was also shown that 'beam-on' data alone were enough to give precisions in range determination better than $1 \mathrm{~mm}$ when at least $5 \mathrm{~Gy}$ was delivered [15].

A crucial issue for a successful application of PET to detect range deviations is reliable Monte Carlo predictions of the expected particle range. From the treatment plan, the time course of the delivery, and the planning CT scan, the activity map and particle range can be predicted in any time-frame. Although analytical approaches can offer a fast solution for this purpose $[23,24]$, Monte Carlo predictions are considered more accurate [25]. The validation of PET modelling against experimental data has been performed in the past with various Monte Carlo generators [2637]. Since beam-background at cyclotrons was considered a major limitation, none of these studies include range measurements performed during target irradiation with a cyclotron. Also, since PET dose verification is generally more relevant for deep-seated tumours, most studies focus on high energies. However, range verification can be desirable also when irradiating more superficially located tumours, such as for instance ocular tumours and head-and-neck tumours.

The scope of this work is to present 'in-beam' PET proton range verifications with data acquired both during and after cyclotron irradiation with $62 \mathrm{MeV}$ protons, and to compare these PET data to Monte Carlo predictions. More precisely, we use a set of PET $\beta^{+}$activity data acquired during and after PMMA phantom irradiation from the CATANA cyclotron, partly reported previously [22], and compare the measured range with those predicted by the FLUKA Monte Carlo generator [38,39]. We investigate what $\beta^{+}$emitting isotopes are formed, and present Monte Carlo predictions and measurements of the proton range under different irradiation conditions. In particular, we will show that range monitoring can be performed also during irradiation, despite the large beam backgrounds. Different to most previous studies, we focus on verifying the proton range, and do not intend to perform a detailed validation of the predicted activity map. This is on one hand because our detector has partial angular coverage only, resulting in image artefacts, and on the other hand because we did not perform a full signal propagation, as would be necessary for this purpose. In contrast to several previous studies performed with FLUKA in this context for protons $[27-29,31,32]$, where proton track length was folded with external experimental cross section data, we have now used directly the prediction of newly developed FLUKA models $[40,41]$. These models have been benchmarked with up-to-date experimental nuclear cross section data. The present study therefore also helps to improve our understanding of the involved nuclear processes.

\section{Methods and materials}

\section{PET system}

We used a planar PET system developed at INFN (National Institute of Nuclear physics, Istituto Nazionale di Fisica Nucleare) and the University of Pisa, previously described in Refs. $[15,22,42,43]$. It consisted of two planar $10 \times 10 \mathrm{~cm}^{2}$ detector heads, each composed of four modules of $5 \times 5 \mathrm{~cm}^{2}$ each, which 
were placed $20 \mathrm{~cm}$ apart. Each module contains $23 \times 23$ blocks of segmented LYSO crystals ( $2.0 \mathrm{~mm}$ pitch, $16 \mathrm{~mm}$ thickness), utilizing multi-anode position sensitive photomultipliers and a fast frontend based on constant fraction discrimination. Data acquisition was performed by an FPGA, which embeds a coincidence processor with a time window of $10 \mathrm{~ns}$. The system was connected to a PC through a USB connection. In Fig. 2 the system is displayed, together with the $x, y$ and $z$-axes. The energy and spatial resolution was measured using a ${ }^{22} \mathrm{Na}$ cubic source of $0.25 \mathrm{~mm}$ dimension. Due to the limited solid angle coverage the resolution of the detector was not uniform along $x, y$, and $z$. In the centre of the field of view (FOV) the energy resolution (full width at half maximum, FWHM) was $18 \%$ at $511 \mathrm{keV}$ and spatial resolution (FWHM) in $x, y$, and $z$ was $7.0,1.8$ and $1.7 \mathrm{~mm}$, respectively, using the reconstruction algorithm as described in Section 2.3. To be able to detect the particle range in tissue, the system was mounted with the $z-y$ plane parallel to the direction of the beam. The system was designed for being installed in the beam-line ("in-beam"), so data could be taken both while the beam is on and while the beam is off, i.e., after treatment.

\section{Phantom irradiation}

PMMA phantoms were irradiated with $62 \mathrm{MeV}$ protons at the CATANA treatment facility [44]. Since 2002 about 300 patients have been treated here for ocular pathologies like uveal melanoma. A Superconducting Cyclotron (SC) was used to produce a $62 \mathrm{MeV}$ proton beam. Range shifters, range modulators and ionization chambers were placed downstream of the scattering system, degrading the proton energy to $58 \pm 0.37 \mathrm{MeV}$. The diameter of the beam was $3.4 \mathrm{~cm}$ at phantom entrance, obtained with a brass collimator. The beam was quasi-parallel, and the beam-intensity profile was rectangular. Typical dose rates for treating ocular tumours are up to $30 \mathrm{~Gy} / \mathrm{min}$, very high compared to other tumours. This is possible in these treatments, thanks to very precise localization of the tumour with the help of tantalum clips placed around the lesion on the outer sclera. In our experiments, protons were impinging on a PMMA target of $5 \times 5 \times 7 \mathrm{~cm}^{3}$ in varying time-frames. The target was fixed inside a PMMA phantom holder. Table 1 summarizes the experimental parameters of the irradiation for the data acquisitions. In the last 4 acquisitions the irradiation time was about 2 min and only the dose rate was varied. These 4 acquisitions were mainly used to

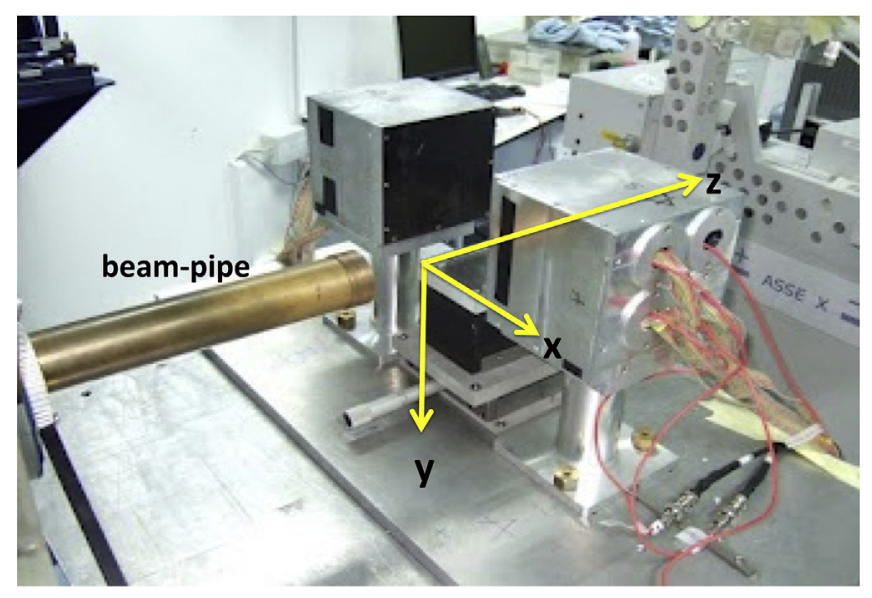

Figure 2. A picture of the experimental setup during data-taking at CATANA, with the reference system indicated.
Table 1

Data acquisitions.

\begin{tabular}{lclc}
\hline Acquisition & Total dose (Gy) & Dose rate (Gy/min) & Irradiation time $(\mathrm{s})$ \\
\hline 1 & 1.1 & 0.69 & 96 \\
2 & 2.1 & 0.67 & 188 \\
3 & 3.7 & 7.8 & 29 \\
4 & 7.5 & 8.7 & 52 \\
5 & 8.0 & 4.2 & 113 \\
6 & 10.0 & 5.0 & 120 \\
7 & 10.0 & 4.9 & 123 \\
8 & 15.0 & 7.7 & 117 \\
\hline
\end{tabular}

verify that the system responded correctly with dose for the same irradiation time. For all data acquisitions the beam-on acquisition time was equal to the beam-off acquisition time, i.e., for an irradiation of $t_{\text {irr }}$ seconds, beam-on events were detected in time-frame $\left[0, t_{\text {irr }}\right]$ and beam-off events were detected immediately following the irradiation in a time-frame $\left[t_{\text {irr, }}, 2 t_{\text {irr }}\right]$.

\section{PET data processing and reconstruction}

PET data were acquired in the form of photons pairs. Each pair containing at least one photon outside the energy window [350, 850] keV was discarded. Data were divided into beam-on and beam-off events, using equal time-frames. Line-of-response (LOR) coincidence events were stored in a sparse format containing the number of hits detected in each LOR. Image reconstruction of the acquired activity was performed using the iterative Maximum Likelihood Expectation Maximization (MLEM) algorithm based on a pre-computed system response model [22]. The total reconstructed field-of-view (FOV) was $10 \times 10 \times 10 \mathrm{~cm}^{3}$ and each voxel of the reconstructed image was $1 \times 1 \times 1 \mathrm{~mm}^{3}$. To account for issues such as non-uniform detector efficiencies, a direct normalization technique was previously implemented using a planar source of $110 \times 110 \times 3 \mathrm{~mm}^{3}$ filled with FDG. The planar source was posed in between the two detector heads in order to illuminate all system LORs. Furthermore, a standard correction for random coincidences was included in the MLEM algorithm. No attenuation correction was performed (see Section 4). More details about the reconstruction procedure can be found elsewhere [22]. The visual assessment of the reconstructed images demonstrated that 5 iterations were sufficient for obtaining a high quality image with acceptable noise level, i.e., no major improvement was seen when using more iterations. Results reported in this work were all based on 5 iterations.

\section{Monte Carlo simulations}

The FLUKA Monte Carlo generator $[38,39]$ was used to simulate the proton interactions and induced activity. FLUKA is a fully integrated particle physics Monte Carlo simulation package with applications in high-energy experimental physics, engineering, medical physics, radiation biology, and many more. In this study we used a development version, Fluka2013 Version 0.0 (5/16/ 2013), with newly updated experimental nuclear interaction cross section data, taken from the available data from the OECD (Organisation for Economic Cooperation and Development) Nuclear Energy Agency Data Bank. In most previous studies using FLUKA [24-29,31,32], FLUKA was used only to calculate the proton track length. The FLUKA predictions were then folded with external experimental cross section data. Here, FLUKA was used as stand-alone framework for simulating the $\beta^{+}$annihilations similar to what was done by Sommerer et al. [30], but now including the 
up-to-date predictions of newly developed FLUKA models [40,41]. Relevant cross sections will be shown in a separate publication by the FLUKA group.

We used the default settings for precision simulations 'PRECISIO'. The beam energy and uncertainty was 58 and $0.37 \mathrm{MeV}$, respectively. The energy threshold for photon production was set to $100 \mathrm{keV}$. We included the phantom geometry and density of the PMMA phantoms directly in FLUKA and did not make use of treatment plans and CT scans. For each annihilation event, we scored the $\beta^{+}$emitting isotope $\left({ }^{11} \mathrm{C},{ }^{15} \mathrm{O}\right.$, etc.), the spatial coordinates and time of the nuclear decays and of the subsequent annihilation, and the photon kinematics. All information was analyzed with the ROOT toolkit [45]. Smearing with a Gaussian distribution was applied to account for the finite detector resolution, where the ${ }^{22} \mathrm{Na}$ measurements (see Section 2.1) were used to determine the smearing parameters. For each data-acquisition in Table 1 we simulated the corresponding Monte Carlo sample, containing $200 \mathrm{M}$ initial protons. We remark that of an initial amount of $200 \mathrm{M}$ protons, there were in total about $1.3 \mathrm{M}$ events with $\beta^{+}$emitters produced and decaying at any time, i.e., about 1 out of 160 events produced a $\beta^{+}$emitter. Finally, it must be noted that the Monte Carlo distributions shown are pure MC truth distributions with photons emitted in a $4 \pi$ geometry, and that no full photon propagation, detector simulation and signal acquisition was included in the simulation (see Section 4).

\section{Data analysis}

We compared one-dimensional activity distributions of Monte Carlo simulations and experimental data. The 1-D activity distribution was obtained by projecting all events with $\left|x^{2}+y^{2}\right|<1 \mathrm{~cm}$ (see also [15]) on the $z$-axis. The activity was expressed in grey values of the images. We determined the $50 \%$ rise and $50 \%$ fall-off position of the 1-D profile with the help of an error function, and evaluated the difference between these, which we called $\Delta$ w50\%. We stress that we do not intend to compare the exact profile shape for two reasons. Firstly, we did not include attenuation corrections in the reconstruction algorithm of the PET data, which is expected to cause an artificial activity maximum at the beam entrance of the phantom, making a direct comparison of the shape inappropriate. Second, our PET detector has incomplete angular coverage. The planar configuration results in a bad resolution along the $x$-axis, causing image reconstruction artifacts and somewhat distorted 1-D $z$-profiles. As discussed in Section 4 and Appendix A, both these issues do not influence the value of $\Delta \mathrm{w} 50 \%$. At present we thus only focus on comparing $\Delta \mathrm{w} 50 \%$, in data and Monte Carlo. Also, we will analyze the relative amounts of activity for the different dataacquisitions for data and Monte Carlo. For the Monte Carlo simulations we investigated in addition which isotopes are involved in beam-on and beam-off $\beta^{+}$activity generation, and studied their 1-D profile shapes and time profiles.

\section{Results}

Monte Carlo predictions of isotope production in beam-on and beam-off acquisition

In Table 2 we display the percentages of the most important isotopes yielding a positron annihilation during 2 min beam-on (3rd column) and 2 min beam-off (4th column) acquisition, which were produced in 2 min of PMMA phantom irradiation. The numbers in Table 2 correspond to acquisition number 6 from Table 1. With 'Others' we mean other processes not included in the isotope list, which lead to a $\beta^{+}$annihilations in the requested time interval. These are mainly annihilations resulting from decays of excited nuclei, emitting a high energetic photon, which leads via electron-positron pair production to a positron annihilation. Table 2 shows that for beam-on data acquisitions, there is a substantial amount of very short-lived isotopes contributing to the activity profile. Although not shown in Table 2, we mention that we observed that the relative amount of short-lived isotopes increases (decreases) for smaller (larger) irradiation times. The time profiles of the various isotopes are given in Fig. 3, where we note the signal rise followed by the exponential decay. Figure 4 shows time profiles of the dominant isotopes in the FLUKA Monte Carlo simulation, i.e., ${ }^{11} \mathrm{C}$ and ${ }^{15} \mathrm{O}$. Figure 5 shows the $1-\mathrm{D}$ activity profiles for the different isotopes, as predicted by FLUKA, in beam-on (blue) and beam-off (red) conditions. From this figure we note that these short-lived isotopes tend to increase slightly the tails of the distribution. Still, useful information can be extracted, and the distal fall-off position increases only very slightly according to the Monte Carlo simulations (see Table 3 described in Section 3.2). We also note that the ${ }^{13} \mathrm{~N}$ spectrum has a characteristic peak at the end of the particle range, explained by the fact that the cross section for ${ }^{13} \mathrm{~N}$ production peaks at low energy, i.e. ${ }^{13} \mathrm{~N}$ is abundantly produced at the end of the projectile range, when it has almost come to rest.

\section{Comparison between data and Monte Carlo predictions}

The 1-D activity profiles for all 8 acquisitions are given in Fig. 6 (beam-on) and Fig. 7 (beam-off), with the determination of the rise and fall-off position. The corresponding 1-D Monte Carlo profile is displayed as well, where the Monte Carlo profiles are scaled with a factor 0.002 (equal for all profiles both beam-on and beam-off) times the corresponding dose, in order to fit inside the same plot as the data profiles. From Figs. 6 and 7 we observe the following. First of all, we notice a clear difference in shape between data and Monte Carlo, which we discuss in Section 4. Secondly, given the fact that one single scaling factor is used, we note that the relative amount of $\beta^{+}$activity is well reproduced by FLUKA in beam-on and beam-off production under all irradiation conditions. Finally, the ratio between beam-on and beam-off activity production is seen to

Table 2

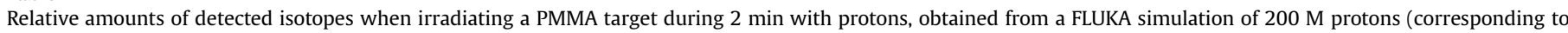
acquisition 6).

\begin{tabular}{|c|c|c|c|}
\hline$\beta^{+}$Emitting isotope & Half-life $\left(t_{1 / 2}\right)$ & Percentage in 2 min beam-on acquisition: $t=[0.120] \mathrm{s}$ & Percentage in 2 min beam-off acquisition $t=[120,240] \mathrm{s}$ \\
\hline${ }^{15} \mathrm{O}$ & $2 \min$ & $50.6 \%$ & $62.4 \%$ \\
\hline${ }^{11} \mathrm{C}$ & $20 \mathrm{~min}$ & $18.1 \%$ & $32.8 \%$ \\
\hline${ }^{8} \mathrm{~B}$ & $770 \mathrm{~ms}$ & $8.4 \%$ & $0.1 \%$ \\
\hline${ }^{10} \mathrm{C}$ & $19 \mathrm{~s}$ & $6.4 \%$ & $1.9 \%$ \\
\hline${ }^{12} \mathrm{~N}$ & $11 \mathrm{~ms}$ & $4.8 \%$ & $0.0 \%$ \\
\hline${ }^{14} \mathrm{O}$ & $71 \mathrm{~s}$ & $0.9 \%$ & $0.8 \%$ \\
\hline${ }^{13} \mathrm{~N}$ & $10 \mathrm{~min}$ & $0.8 \%$ & $1.3 \%$ \\
\hline Others & & $10.1 \%$ & $0.7 \%$ \\
\hline
\end{tabular}



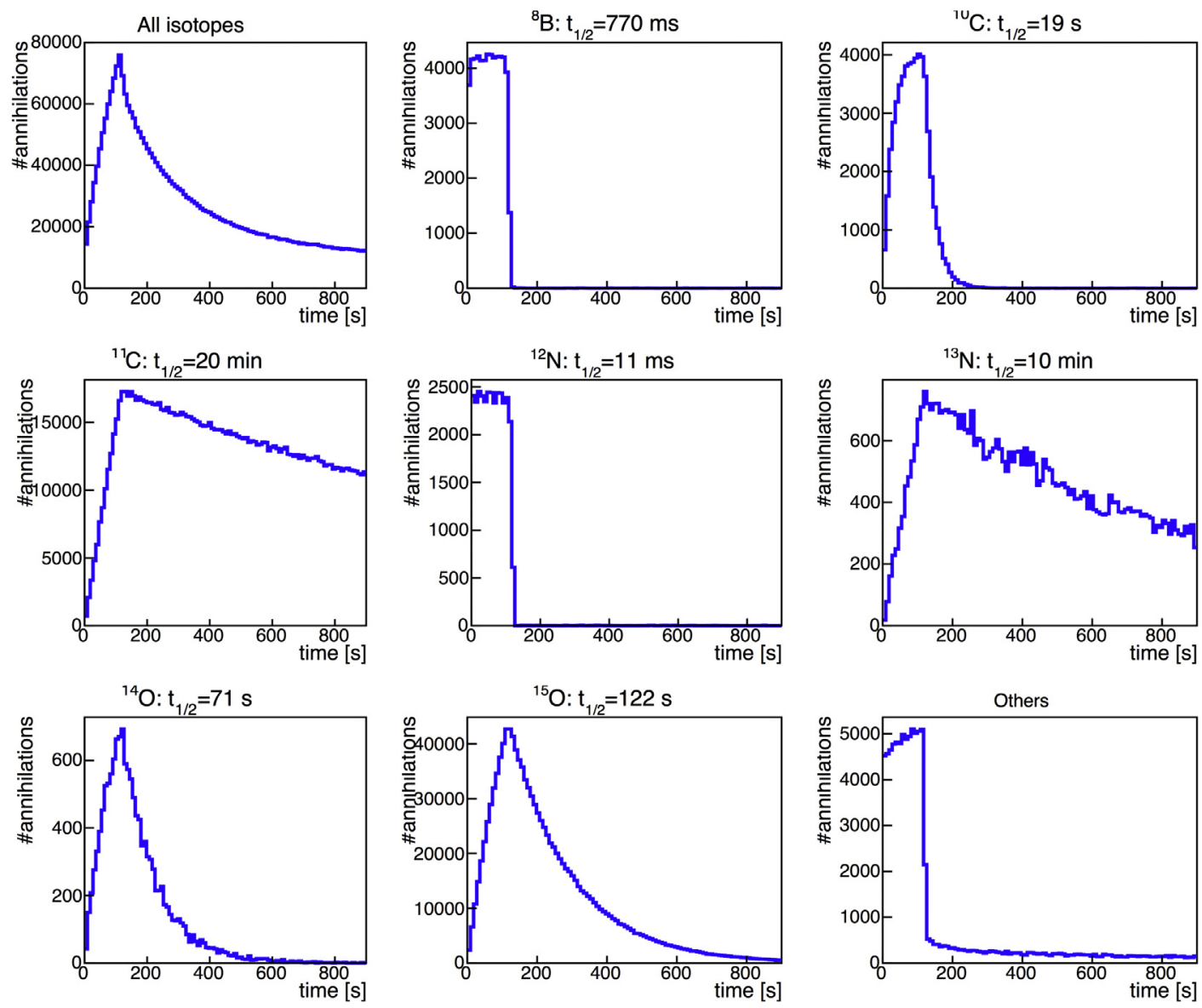

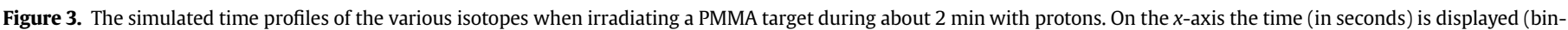

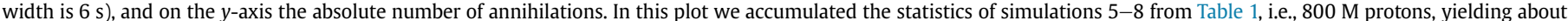
$5 \mathrm{M}$ annihilations.

be roughly 1 in both Monte Carlo and data, independent of the irradiation time (see below).

Table 3 displays the values for $\Delta \mathrm{w} 50 \%$, the distance between $50 \%$ distal fall-off and $50 \%$ rise positions, for these data acquisitions. Despite the differences in profile shape, Table 3 shows that

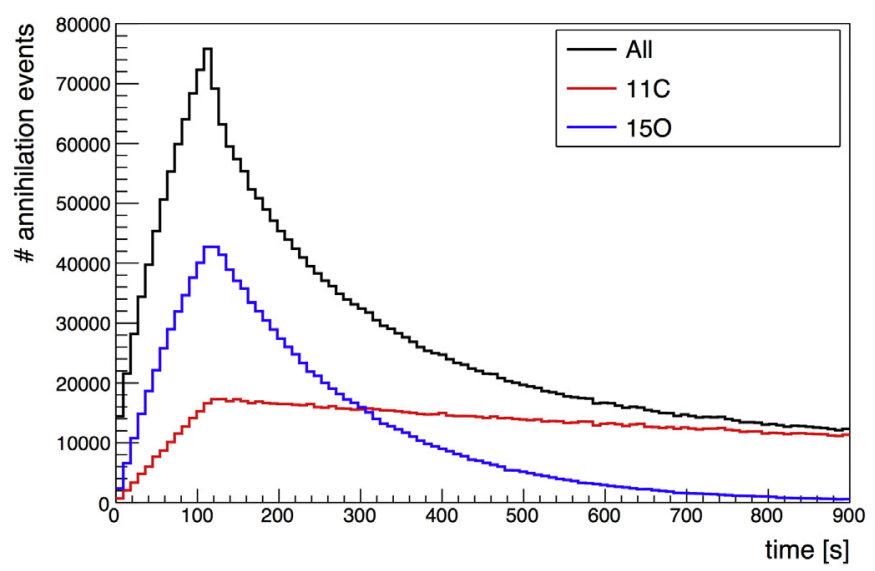

Figure 4. The simulated time profiles of the two most important isotopes, ${ }^{15} \mathrm{O}$ an ${ }^{11} \mathrm{C}$ for irradiation of a PMMA target during 2 min with protons. We display on the $x$-axis the time (in seconds, bin-width is $9 \mathrm{~s}$ ), and on the $y$-axis the absolute number of annihilations. In this plot we again accumulated the statistics of simulations 5-8 from Table 1. there is a good agreement in $\Delta \mathrm{w} 50 \%$ between data and Monte Carlo. For all irradiation times and doses considered, the difference between Monte Carlo and data in $\Delta \mathrm{w} 50 \%$ is less than $1 \mathrm{~mm}$ for PMMA phantoms, for both beam-on and beam-off conditions. Table 3 also shows that both in data and MC the distal fall-off position is slightly larger for beam-on than for beam-off acquisitions: the difference in $\Delta \mathrm{w} 50 \%$ between beam-on and beam-off conditions is 0.3 and $0.1 \mathrm{~mm}$ for data and Monte Carlo, respectively.

Figure 8 shows the area of all profiles for both the data and Monte Carlo profiles shown in Figs. 6 and 7. Firstly, we see a good linearity between the area of the data profile and the dose for the 4 data acquisitions taken with equal irradiation time $(8,10,10$, $15 \mathrm{~Gy}$ ), showing that up to the highest dose rate of these (7.5 Gy/ min) no saturation occurs. Secondly, we note that this linearity does not hold for the other acquisitions with different irradiation times. This is explained by the different isotopes that are involved in producing $\beta^{+}$annihilations: the shorter the irradiation timeframe, the more important is the contribution of the shorter lived isotopes, and vice-versa. Thus, the relative amount of $\beta^{+}$ emitting isotopes, which are produced and subsequently decay, depends on the irradiation time. In fact, the same behaviour holds for the Monte Carlo simulations, as can be seen in Fig. 8, where the Monte Carlo predictions are also reported. From Fig. 8 we see a very good agreement between data and Monte Carlo in the relative amount of produced activity in different time-frames, both for beam-on and beam-off conditions. 

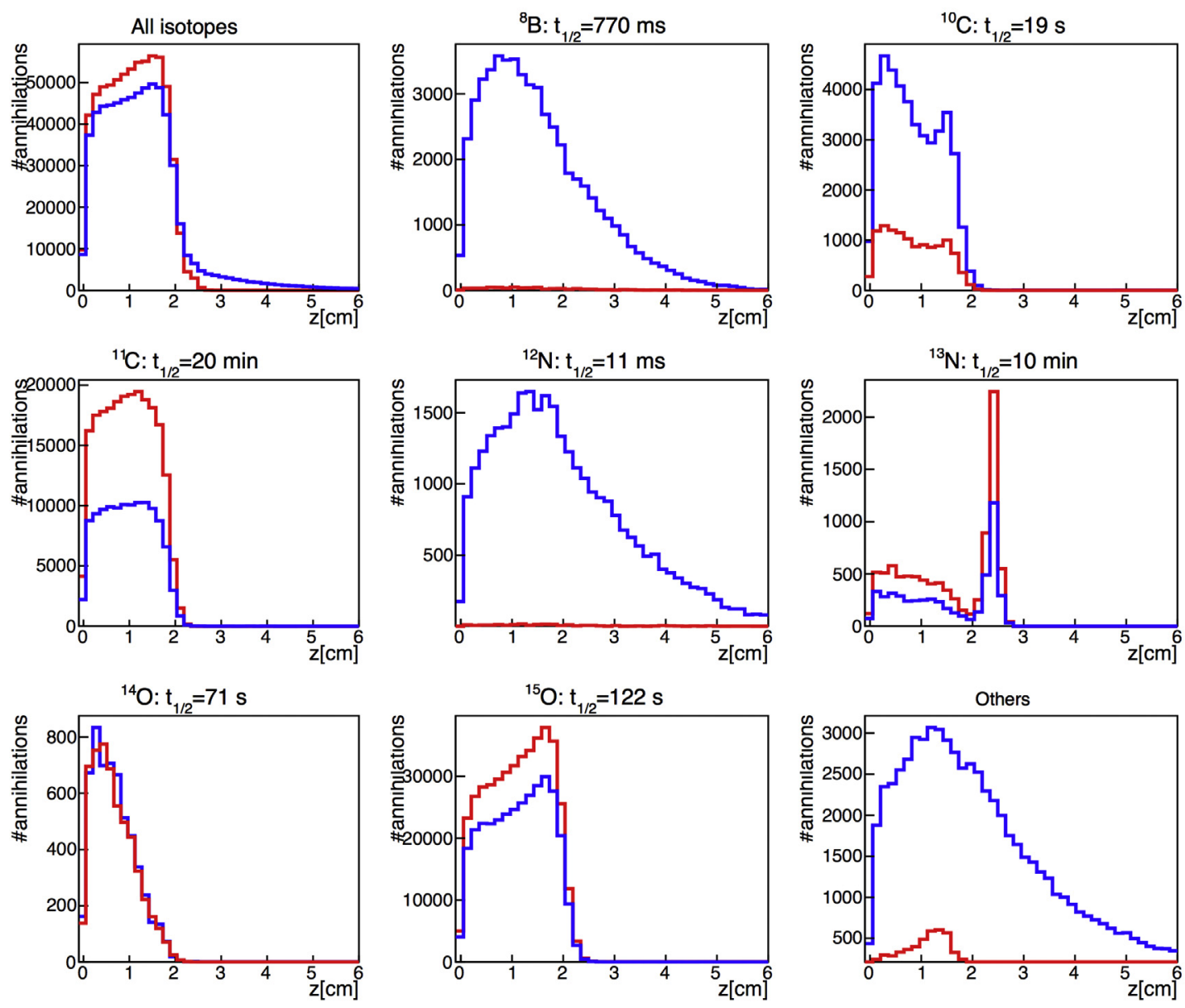

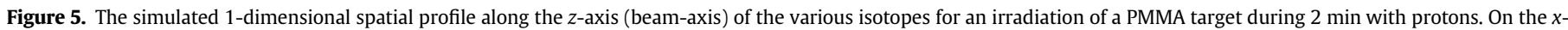

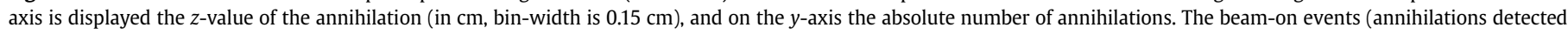

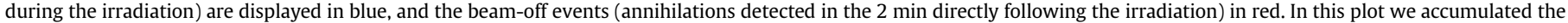
statistics of the simulations 5 to 8 from Table 1. (For interpretation of the references to colour in this figure legend, the reader is referred to the web version of this article.)

Figure 9 shows the ratio between the amount of beam-on and beam-off production for the 8 data acquisitions. The ratio is about 1 both for data and Monte Carlo. Thus, the beam-on events provide a substantial amount of statistics in addition to beam-off events.

\section{Discussion}

Table 3 demonstrates that the proton range measurements, represented by $\Delta \mathrm{w} 50 \%$, correspond well to the predictions of the FLUKA Monte Carlo generator, both in beam-on and beam-off

Table 3

Data and Monte Carlo predictions for the value of $\Delta \mathrm{w} 50 \%$ for the 8 acquisitions.

\begin{tabular}{|c|c|c|c|c|c|}
\hline \multirow[t]{2}{*}{ Acquisition } & \multirow{2}{*}{$\begin{array}{l}\text { Irradiation } \\
\text { time }(\mathrm{s})\end{array}$} & \multicolumn{2}{|c|}{$\Delta \mathrm{w} 50 \%(\mathrm{~mm})$ beam-on } & \multicolumn{2}{|c|}{$\Delta \mathrm{w} 50 \%(\mathrm{~mm})$ beam-off } \\
\hline & & Data & MC & Data & MC \\
\hline 1 & 96 & 18.7 & 19.6 & 18.6 & 19.5 \\
\hline 2 & 188 & 19.4 & 19.6 & 19.0 & 19.3 \\
\hline 3 & 28 & 20.0 & 19.4 & 18.4 & 19.4 \\
\hline 4 & 52 & 19.4 & 19.5 & 18.6 & 19.5 \\
\hline 5 & 113 & 19.2 & 19.6 & 19.0 & 19.4 \\
\hline 6 & 120 & 19.2 & 19.6 & 18.9 & 19.5 \\
\hline 7 & 123 & 19.4 & 19.6 & 19.0 & 19.4 \\
\hline 8 & 117 & 19.2 & 19.6 & 18.9 & 19.4 \\
\hline
\end{tabular}

conditions, for various irradiation conditions. Despite the large beam background, we have shown that valuable range measurements can be performed not only after, but also during proton irradiation.

We have quantified the error on $\Delta \mathrm{w} 50 \%$ from the fitting procedure in the Monte Carlo simulations and in data. By varying the fitting ranges we found that the error due to the fitting procedure in Monte Carlo and data is below $0.3 \mathrm{~mm}$. The error from Monte Carlo statistics is negligible compared to other errors, thanks to the very high statistics samples (200 M) we used for each acquisition. For determining the statistical error in $\Delta \mathrm{w} 50 \%$ in data we would have to take many data acquisitions under the same conditions. Given the limited amount of beam-time available to us we have not performed such data acquisitions. The systematic errors on $\Delta w 50 \%$ in Monte Carlo and data have not been determined at this stage, but will be part of a future study.

Differences in 1-D profile shape between Monte Carlo simulations and data were observed. In particular, we observe a maximum in the profile at phantom entrance. Although the scope of the present paper was only to present comparisons of $\Delta \mathrm{w} 50 \%$ rather than to validate the whole activity distributions, let's discuss what we believe causes these differences.

- Absence of attenuation corrections in data. Since photons at the phantom entrance are not attenuated, a signal increase is 

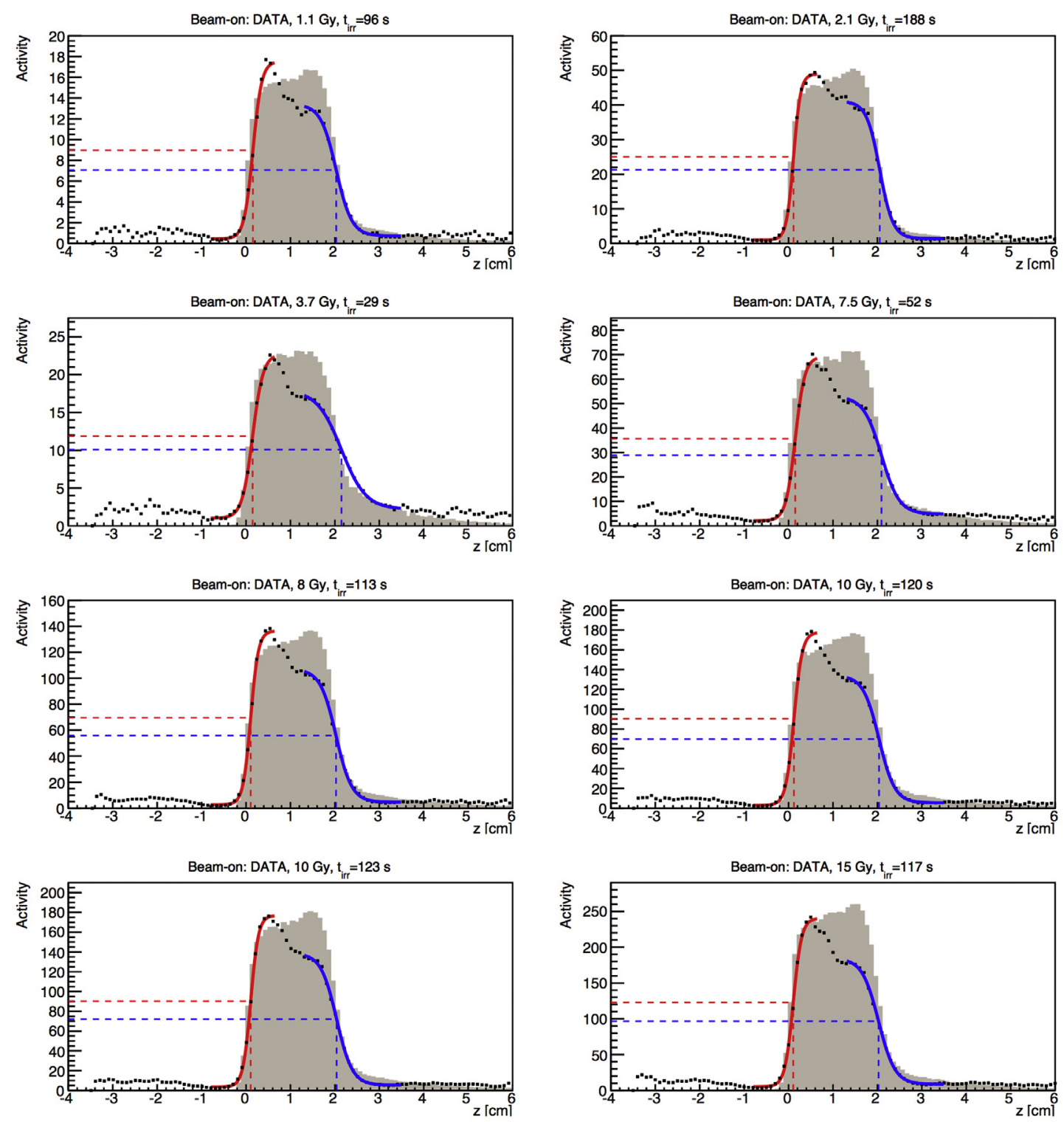

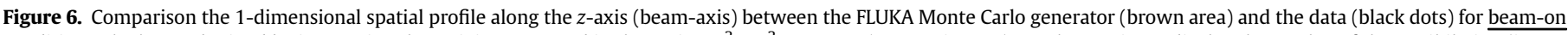

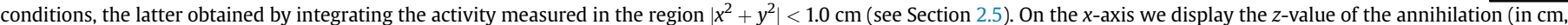

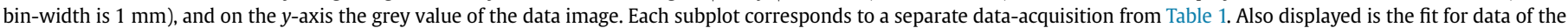

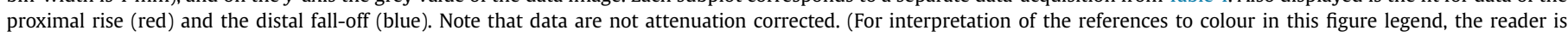
referred to the web version of this article.)

expected at the phantom entrance. To understand the impact of the attenuation effect better, we performed MC simulations with the GATE [46] Monte Carlo simulator of cylinders filled with FDG (partly) surrounded by PMMA. We noted that the profile shape was strongly influenced by attenuation effects. In fact, the simulations strongly indicate that the maximum in the 1-D activity profiles, which we observed in data, is caused by attenuation effects in the phantom and phantom holder. However, the value of $\Delta w 50 \%$ was not significantly influenced by attenuation. More details are given in Appendix A1. As a sideremark, we mention that an increase at phantom entrance was observed also in data taken at CNAO for the energy range 93-112 MeV, where range measurements were also found to agree very well with Monte Carlo predictions [42]. This also suggests that attenuation corrections are not strictly necessary for proton range verifications.

- Incomplete angular coverage of our planar detector. This is known to cause serious image artifacts and varying resolution in the FOV, rendering these type of detectors inadequate for a direct comparisons of the profile shape between data and Monte Carlo. In order to understand better the geometrical effects, and to be sure that the detector response was as expected, we acquired activity data of cylinders filled with FDG placed at various positions, and determined the value of $\Delta \mathrm{w} 50 \%$. These data were compared with GATE Monte Carlo simulations, including full photon propagation. A good agreement between data and Monte Carlo simulations was found in shape and in $\Delta \mathrm{w} 50 \%$, as is shown in more detail in Appendix A2. This demonstrates that the distal fall-off and rise positions can be measured accurately despite the incomplete angular coverage of our detector.

To compare the FLUKA Monte Carlo simulations directly to the acquired data, a full propagation of the photons in the Monte Carlo simulations would have been required, including a simulation for the detector and the electronics, which should be passed through the same reconstruction algorithm as the data. A 

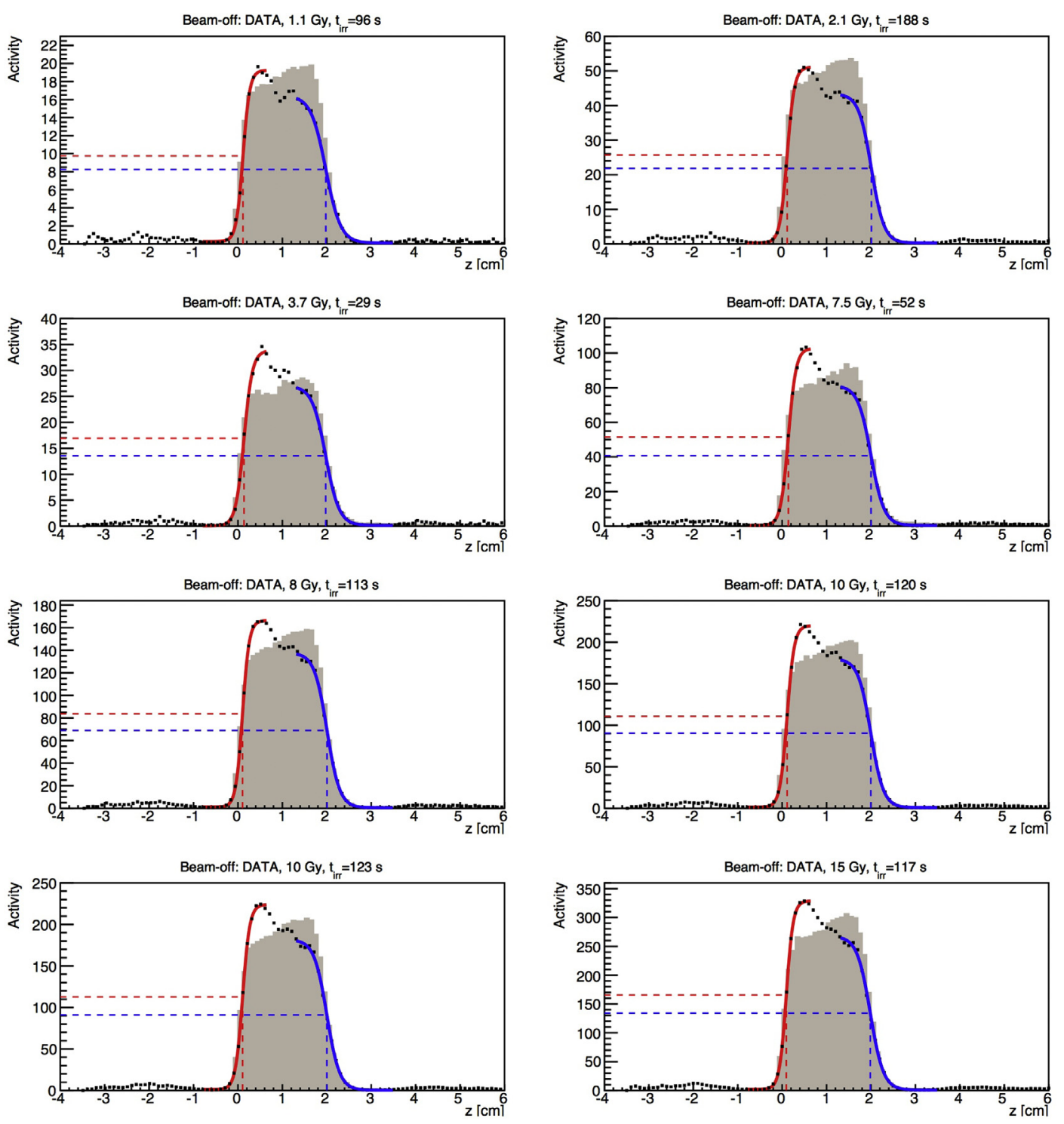

Figure 7. The same as Fig. 6, but now for beam-off conditions. Note that data are not attenuation corrected.

preliminary study performed with FLUKA, where the photons were propagated to the detector planes, and the hit positions subsequently passed to the reconstruction software, confirmed that the maximum at entrance is caused by attenuation and geometrical effects. However, the amount of simulated proton statistics necessary for a full simulation study requires large amounts of CPU and storage space, and is beyond the scope of the current work. For future studies we plan to include the response function of the detector so that the geometrical influences and attenuation effects in the phantom can be simulated.

Another drawback of our study is that only a relative comparison between data and Monte Carlo has been done. By doing a careful comparison of the response of the system to sources of accurately known activity, a more quantitative analysis should be feasible. As described above, the relative response of the system is correct and corresponds well with the FLUKA Monte Carlo.

In this study we have compared the distance between the $50 \%$ level of the proximal rise and distal fall-off positions. We are aware that the $50 \%$ level of the distal fall-off position is only one of the possible criteria for monitoring the range of protons. Lower thresholds may be used [2], but may be more sensitive to noise. Given the low statistics of the datasets involved in these studies we have chosen to investigate only the $50 \%$ level.

We are currently improving the Monte Carlo simulations by adding full propagation of the photons in FLUKA, so we can correctly simulate geometrical and attenuation effects. Also, effort is being put into speeding up the FLUKA simulations. Moreover we plan to implement attenuation corrections into the reconstruction software. On the hardware side, we are currently enlarging the PET detector. We plan to include more experimental data for different materials in the near future, and do a full comparison between data and Monte Carlo simulations. For this purpose we intend to perform more data acquisitions for homogeneous, inhomogeneous and anthropomorphic phantoms at the higher energy hadron therapy facility in Italy, CNAO [47].

\section{Conclusion}

In this work we showed a set of PET activity data taken during and after irradiation of PMMA phantoms with $62 \mathrm{MeV}$ protons at 

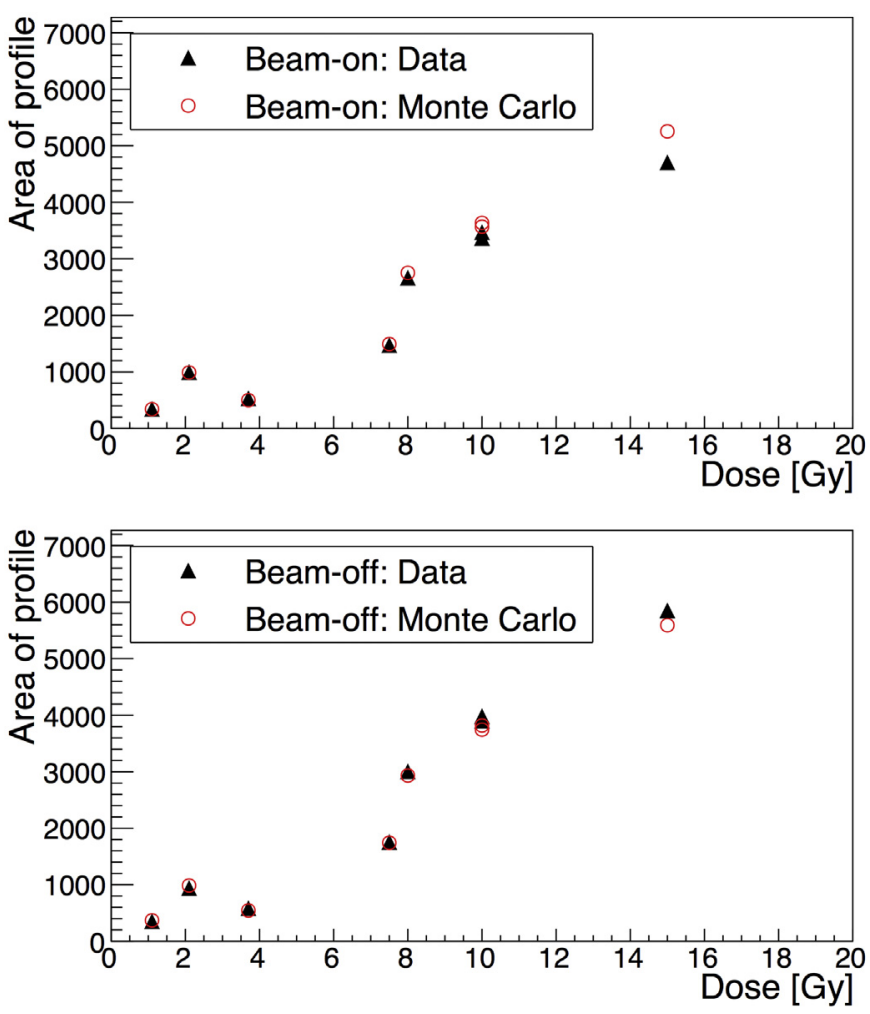

Figure 8. Top: the area of the one dimensional $z$-profiles of the 8 data acquisitions displayed in Fig. 6 (beam-on) as function of the delivered dose, for data (black triangle) and FLUKA Monte Carlo generator (red open circle). Bottom: the same, but now for the 8 data acquisitions displayed in Fig. 7 (beam-off). (For interpretation of the references to colour in this figure legend, the reader is referred to the web version of this article.)

the CATANA cyclotron facility. We compared measurements of the distance $\Delta \mathrm{w} 50 \%$ between the $50 \%$ distal fall-off and $50 \%$ proximal rise, with predictions from the FLUKA Monte Carlo generator. A good agreement $\Delta \mathrm{w} 50 \%$ was found between data and Monte Carlo simulations. Differences were found to be less than one $\mathrm{mm}$ in various irradiation conditions, including high dose rates and large beam backgrounds.

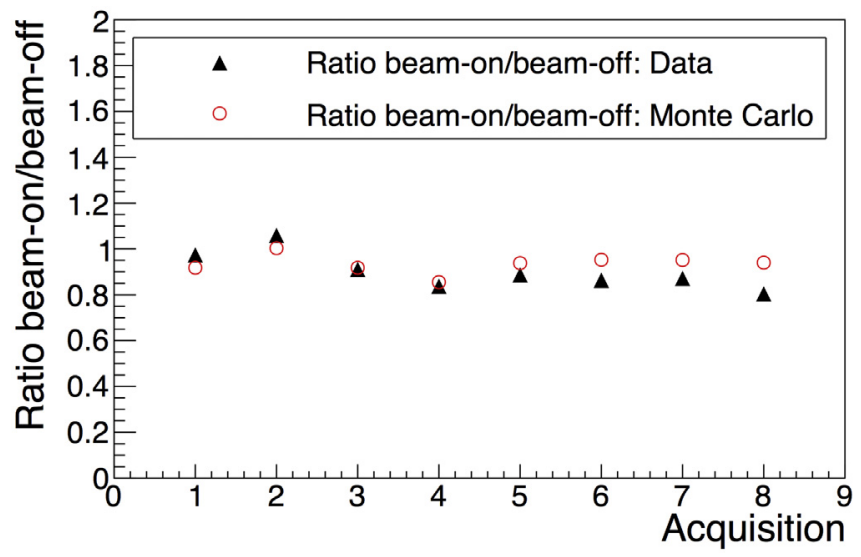

Figure 9. The ratio between the amount of measured beam-on and beam-off activity, obtained by dividing the area of the 1-D profiles from 2 min beam-on (Fig. 6) and 2 min beam-off (Fig. 7) data acquisitions.

\section{Acknowledgements}

This research has been supported by the FULLBEAM-300838 Marie Curie Intra European Fellowship within the 7th European Community Framework Programme, by the INFN-RT 60141 POR CRO FSE 2007-2013 fellowship, and by the FP7-ENVISION project.

\section{Appendix A}

Below we investigate the response function of our PET detector with respect to attenuation effects in the phantom (Section A1) and geometrical influence (Section A2).

\section{A1. Attenuation effects}

We estimated the effect of photon attenuation with the help of Monte Carlo simulations performed with the GATE (Geant 4 Application for Tomographic Emission) Monte Carlo simulator [46], a platform developed to simulate nuclear medicine experimental configurations. This was including full photon propagation, a detector simulation, and reconstruction. We compared a simulation of an active region, which was surrounded by PMMA on all but the left side (Fig. A1 left, "Left side not attenuated"), to a simulation where the active region was embedded on all sides by PMMA (Fig. A1 middle). The FDG cylinder has a diameter of $34 \mathrm{~mm}$ and a height of $2 \mathrm{~cm}$ and was embedded in a phantom and phantom holder. The setup at the left side of Fig. A1 is very similar to the setup at the CATANA data acquisitions, with similar size of the activated region. Figure A1 (right) shows the resulting reconstructed activity profiles for both cases. From this figure it becomes clear that the shape reconstructed in our detector depends strongly on the attenuation of the photons in the phantom. Moreover, this figure strongly suggests that the maximum at beam entrance observed in our data profiles is caused by attenuation effects in the phantom.

\section{A2. Geometrical effects due to limited angular coverage}

In order to understand the geometrical response of the detector and to assure that our detector measured correct values for $\Delta \mathrm{w} 50 \%$, we acquired PET data using a phantom with a cavity filled with FDG (see Fig. A2). Although the activity origin is very different (in one case the activity is generated from nuclear reactions in the PMMA phantom, while in the other we have a positron emitter like $18-\mathrm{F}$ ), the detector response is similar. As can be seen in Fig. A2, we used a cylindrical PMMA phantom, with a cylindrical cavity (diameter $15 \mathrm{~mm}$ and $30 \mathrm{~mm}$ along $z$, the beam direction) filled with FDG and placed in various positions. The acquired data were compared with Monte Carlo simulations from GATE [46] just like in Section A1, including full photon transport. In Fig. A3 we show the activity profiles as function of the $z$-position along the PET planes for 1$)$ the true active FDG region $(30 \mathrm{~mm}$, grey area), 2) the GATE Monte Carlo simulation including full photon propagation (red line, in the web version), and 3) the PET data (black dots). From Fig. A3 it is noticeable that the shape in data is somewhat distorted, and that geometrical effects play a role in the shape of the profile. However, although the shapes change, the distance between the $50 \%$ rise and $50 \%$ fall-off position, $\Delta \mathrm{w} 50 \%$, is about $30 \mathrm{~mm}$ in all cases, reproducing very well the true depth of the hot cavity used in these experiments. Thus, we are confident that our detector gives the correct result for $\Delta \mathrm{w} 50 \%$, despite the limited angular coverage. 


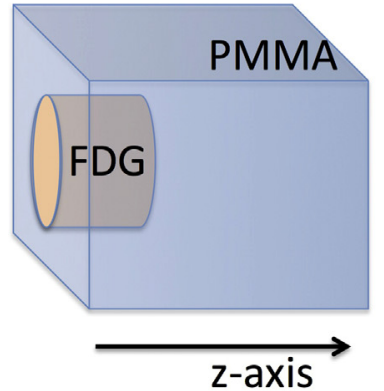

Left side not attenuated
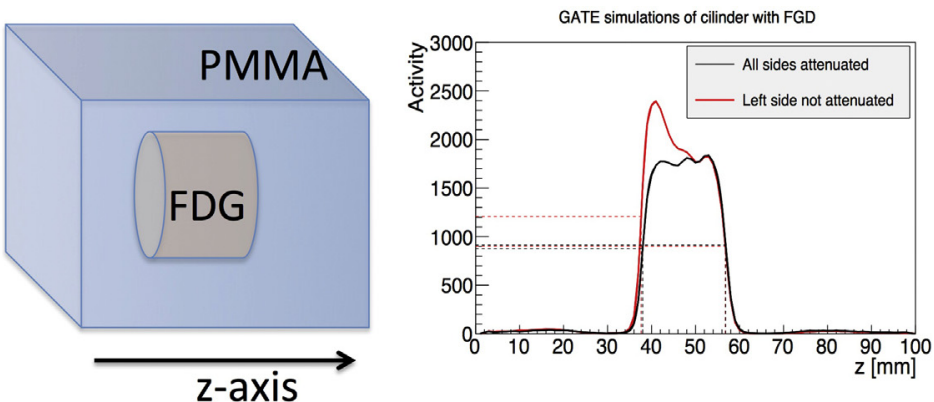

\section{All sides attenuated}

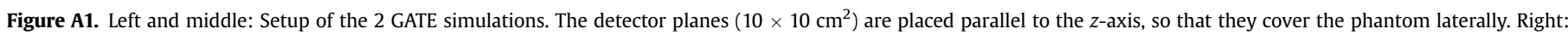
resulting simulated activity profiles from the GATE Monte Carlo generator.

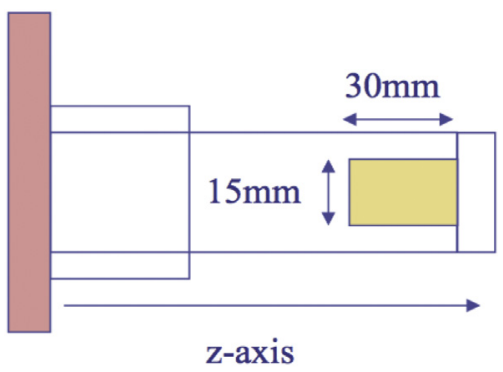

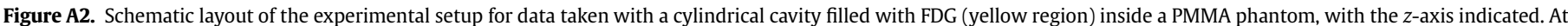
the left it is connected to a pc-steered translator. (For interpretation of the references to colour in this figure legend, the reader is referred to the web version of this article.)
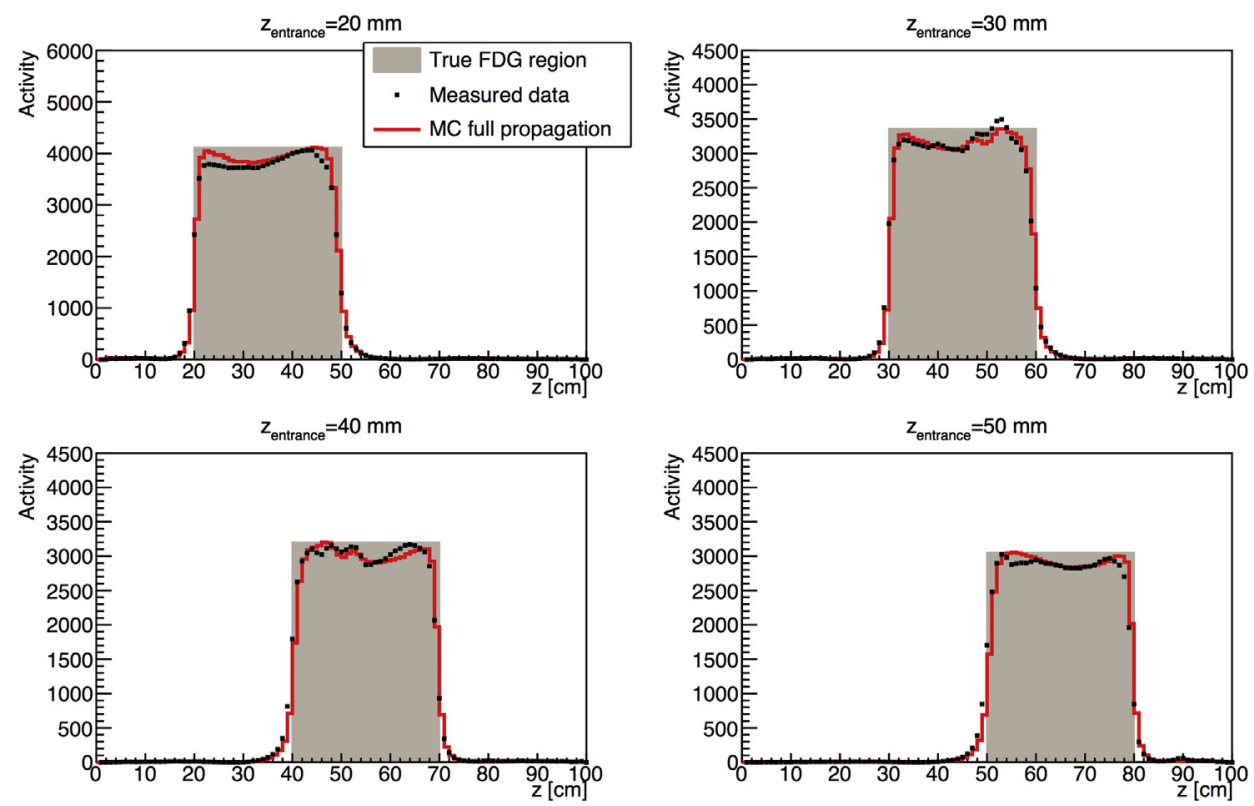

Figure A3. Example of 1-D activity profiles in various positions. The true active region was here normalized to the maximum of the GATE MC profile.

\section{References}

[1] Loeffler JS, Durante M. Charged particle therapy optimization, challenges and future directions. Nat Rev Clin Oncol 2013;10:411-24.

[2] Knopf AC, Lomax A. In vivo proton range verification: a review. Phys Med Biol 2013;58(15):R131-60.

[3] Zhu X, El Fahkri G. Proton therapy verification with PET imaging. Theranostics 2013;3(10):731-40.
[4] Parodi K, Paganetti H, Shih HA, Michaud S, Loeffler JS, DeLaney TF, et al. Patient study of in-vivo verification of beam delivery and range, using positron emission tomography and computed tomography imaging after proton therapy. Int J Radiat Oncol Biol Phys 2007;68:920-34.

[5] Knopf AC, Parodi K, Paganetti H, Bortfeld T, Daartz J, Engelsman M, et al. Accuracy of proton beam range verification using post-treatment positron emission tomography/computed tomography as function of treatment site. Int J Radiat Oncol Biol Phys 2011;79:297-304. 
[6] Nishio T, Miyatake A, Inoue K, Gomi-Miyagishi T, Kohno R, Kameoka S, et al Experimental verification of proton beam monitoring in a human body by use of activity image of positron-emitting nuclei generated by nuclear fragmentation reaction. Radiol Phys Technol 2008;1(1):44-54.

[7] Hsi WC, Indelicato DJ, Vargas C, Duvvuri S, Li Z, Palta J. In vivo verification of proton beam path by using post-treatment PET/CT imaging. Med Phys 2009;36(9):4136-46.

[8] Bauer J, Unholtz D, Sommerer F, Kurz C, Haberer T, Herfarth K, et al. Implementation and initial clinical experience of offline PET/CT-based verification of scanned carbon ion treatment. Radiother Oncol 2013;107:218-26.

[9] Zhu X, España S, Daartz J, Liebsch N, Ouyang J, Paganetti H, et al. Monitoring proton radiation therapy with in-room PET imaging. Phys Med Biol 2011:56(13):4041-57.

[10] Min CH, Zhu X, Winey BA, Grogg K, Testa M, El Fakhri G, et al. Clinical application of in-room positron emission tomography for in vivo treatment monitoring in proton radiation therapy. Int J Radiat Oncol Biol Phys 2013;86(1):183-9.

[11] Enghardt W, Parodi K, Crespo P, Fiedler F, Pawelke J, Pönisch F. Dose quantification from in-beam positron emission tomography. Radiother Onco 2004;73(Suppl. 2):S96-8.

[12] Iseki Y, Kanai T, Kanazawa M, Kitagawa A, Mizuno H, Tomitani T, et al. Range verification system using positron emitting beams for heavy-ion radiotherapy. Phys Med Biol 2004:49(14):3179-95.

[13] Nishio T, Miyatake A, Ogino T, Nakagawa K, Saijo N, Esumi H, et al. The development and clinical use of a beam ON-LINE PET system mounted on a rotating gantry port in proton therapy. Int J Radiat Oncol Biol Phys 2010;76(1):277-86.

[14] Miyatake A, Nishio T, Ogino T, Saijo N, Esumi H, Uesaka M. Measurement and verification of positron emitter nuclei generated at each treatment site by target nuclear fragment reactions in proton therapy. Med Phys 2010;37(8): 4445-55.

[15] Sportelli G, Belcari N, Camarlinghi N, Cirrone GA, Cuttone G, Ferretti S, et al First full-beam PET acquisitions in proton therapy with a modular dual-head dedicated system. Phys Med Biol 2014;59(1):43-60.

[16] Enghardt W, Crespo P, Fiedler F, Hinz R, Parodi K, Pawelke J, et al. Charged hadron tumour therapy monitoring by means of PET. Nucl Instrum Methods A 2004;525:284-8.

[17] Crespo P, Shakirin G, Fiedler F, Enghardt W, Wagner A. Direct time-of-flight for quantitative, real-time in-beam PET: a concept and feasibility study Phys Med Biol 2007;52:6795-811.

[18] Yamaya T, Yoshida E, Inaniwa T, Sato S, Nakajima Y, Wakizaka $H$, et al Development of a small prototype for a proof-of-concept of OpenPET imaging. Phys Med Biol 2011;56(4):1123-37.

[19] Tashima H, Yamaya T, Yoshida E, Kinouchi S, Watanabe M, Tanaka E. A singlering OpenPET enabling PET imaging during radiotherapy. Phys Med Biol 2012;57:4705-18.

[20] Parodi K, Bortfeld T, Haberer T. Comparison between in-beam and offline positron emission tomography imaging of proton and carbon ion therapeutic irradiation at synchrotron- and cyclotron-based facilities. Int J Radiat Oncol Biol Phys 2008;71(3):945-56.

[21] Crespo P, Barthel T, Frais-Kolbl H, Griesmayer E, Heidel K, Parodi K, et al Suppression of random coincidences during in-beam PET measurements at ion beam radiotherapy facilities. IEEE Trans Nucl Sci 2005;52(4):980-7.

[22] Camarlinghi N, Sportelli G, Battistoni G, Belcari N, Cecchetti M, Cirrone GAP, et al. An in-beam PET system for monitoring ion-beam therapy: test on phantoms using clinical $62 \mathrm{MeV}$ protons. JINST 2014;9:C04005.

[23] Parodi K, Bortfeld T. A filtering approach based on Gaussian-powerlaw convolutions for local PET verification of proton radiotherapy. Phys Med Biol 2006;51(8):1991-2009.

[24] Attanasi F, Knopf A, Parodi K, Paganetti H, Bortfeld T, Rosso V, et al. Extension and validation of an analytical model for in vivo PET verification of proton therapy: a phantom and clinical study. Phys Med Biol 2011:56(16):5079-98.

[25] Paganetti H. Range uncertainties in proton therapy and the role of Monte Carlo simulations. Phys Med Biol 2012;57(11):R99-117.
[26] Seravalli E, Robert C, Bauer J, Stichelbaut F, Kurz C, Smeets J, et al. Monte Carlo calculations of positron emitter yields in proton radiotherapy. Phys Med Biol 2012:57(6):1659-73.

[27] Parodi K, Ferrari A, Sommerer F, Paganetti H. Clinical CT-based calculations of dose and positron emitter distributions in proton therapy using the FLUKA Monte Carlo code. Phys Med Biol 2007;52(12):3369-87.

[28] Parodi K, Paganetti H, Cascio E, Flanz JB. PET/CT imaging for treatment verification after proton therapy: a study with plastic phantoms and metallic implants. Med Phys 2002;34(2):419-35.

[29] Sommerer F, Parodi K, Ferrari A, Poljanc K, Enghardt W, Aiginger H. Investigating the accuracy of the FLUKA code for transport of therapeutic ion beams in matter. Phys Med Biol 2006;51:4385-98.

[30] Sommerer F, Cerutti F, Parodi K, Ferrari A, Enghardt W, Aiginger H. In-beam PET monitoring of mono-energetic ${ }^{16} \mathrm{O}$ and ${ }^{12} \mathrm{C}$ beams: experiments and FLUKA simulations for homogeneous targets. Phys Med Biol 2009;54(13): 3979-96.

[31] Parodi K, Enghardt W, Haberer T. In-beam PET measurements of $\beta+$ radioactivity induced by proton beams. Phys Med Biol 2002;47:21-36.

[32] Bauer J, Unholtz D, Kurz C, Parodi K. An experimental approach to improve the Monte Carlo modelling of offline PET/CT-images of positron emitters by scanned proton beams. Phys Med Biol 2013;58:5193-213.

[33] Pshenichnov I, Mishustin I, Greiner I. Distributions of positron-emitting nuclei in proton and carbon-ion therapy studied with GEANT4. Phys Med Biol 2006;51:6099-112.

[34] Pawelke J, Enghardt W, Haberer T, Hasch BG, Hinz R, Kramer M, et al. In-beam PET imaging for the control of heavy-ion tumour therapy. IEEE Trans Nucl Sci 1997:2:1099-103.

[35] Paganetti H, Gottschalk B. Test of GEANT3 and GEANT4 nuclear models for $160 \mathrm{MeV}$ protons stopping in $\mathrm{CH}_{2}$. Med Phys 2003;30(7):1926-31.

[36] Del Guerra A, Di Domenico G, Mukhopadhayay D. PET dosimetry in proton radiotherapy: a Monte Carlo study. Appl Radiat Isot 1997;48(10-12): 1617-24.

[37] Oelfke U, Lam GK, Atkins MS. Proton dose monitoring with PET: quantitative studies in Lucite. Phys Med Biol 1996:41(1):177-96.

[38] Battistoni G, Cerutti F, Fassò A, Ferrari A, Muraro S, Ranft J, et al. The FLUKA code: description and benchmarking. In: Proceedings of the hadronic shower simulation workshop 2006, Fermilab, 2006, AIP proceedings 896; 2007. pp. 31-49.

[39] Ferrari A, Sala PR, Fasso A, Ranft J. FLUKA: a multi-particle transport code, CERN-2005-10; 2005. INFN/TC_05/11, SLAC-R-773.

[40] Böhlen TT, et al. The FLUKA code: developments and challenges for high energy and medical applications. In: International conference on nuclear data for science and technology; 2013. in press.

[41] Robert C, Dedes G, Battistoni G, Böhlen TT, Buvat I, Cerutti F, et al. Distributions of secondary particles in proton and carbon-ion therapy: a comparison between GATE/Geant4 and FLUKA Monte Carlo codes. Phys Med Biol 2013;58(9):2879-99.

[42] Rosso V, Battistoni G, Belcaria N, Camarlinghi N, Ferrari A, Ferretti S, et al A new PET prototype for proton therapy: comparison of data and Monte Carlo simulations. J Inst 2013;8:C03021.

[43] Attanasi F, Belcari N, Camarda M, Cirrone GAP, Cuttone G, Del Guerra A, et al Preliminary results of an in-beam PET prototype for proton therapy. Nucl Instrum Methods A 2008;591(1):296-9.

[44] Cirrone GAP, Cuttone G, Lojacono PA, Lo Nigro S, Mongelli V, Patti IV, et al. A $62 \mathrm{Mev}$ proton beam for the treatment of ocular melanoma at Laboratori Nazionali del Sud-INFN (Catania). IEEE Nucl Sci Symp Conf Rec 2003;5: 3658-62.

[45] ROOT toolkit. See http://root.cern.ch/, version April 2013.

[46] Jan S, Santin G, Strul D. GATE: a simulation toolkit for PET and SPECT. Phys Med Biol 2004;49:4543-61.

[47] S. Rossi on behalf of the CNAO collaboration. the status of CNAO. Eur Phys ] Plus 2011;126:78. 